\title{
SEROPREVALENCIA DE HEPATITIS VIRAL B EN ESTUDIANTES UNIVERSITARIOS EN ABANCAY, PERÚ
}

\author{
Max Carlos Ramírez-Soto ${ }^{1, a}$, Milagros Huichi-Atamari²,a, Elsa Gladys Aguilar-Ancori',a, \\ Justo Darwin Pezo-Ochoa 3 ,a
}

\begin{abstract}
RESUMEN
Para determinar la prevalencia de marcadores serológicos de hepatitis viral B en estudiantes universitarios de la ciudad de Abancay, realizamos un estudio transversal en 240 estudiantes de tres universidades, entre enero a octubre de 2010. Previo consentimiento informado, se llenó, por cada estudiante, una ficha epidemiológica y se tomó una muestra sanguínea para determinar la presencia de $\mathrm{HBsAg}$, anti-HBcAg total, anti-HBe, $\mathrm{HBeAg}$ e IgM anti-HBc por el método de ELISA. Se encontró una prevalencia de $2,5 \%$ (seis seropositivos) para el HBsAg y $28,3 \%$ (68 seropositivos) para los anticuerpos Anti-HBcAg. El sexo masculino estuvo asociado con la presencia del anti-HBcAg (OR = 2,0; IC $95 \%, 1,2-$ $3,6)$. No se encontró la presencia del HBeAg e IgM anti-HBc; los seis portadores del HBsAg fueron anti-HBe positivos. En conclusión, la infección por hepatitis B sigue siendo un problema de salud pública en Abancay, con una prevalencia importante en estudiantes universitarios.
\end{abstract}

Palabras clave: Estudios seroepidemiológicos; Hepatitis B; Estudiantes; Abancay; Perú (fuente: DeCS BIREME).

\section{SEROPREVALENCE OF VIRAL HEPATITIS B IN UNIVERSITY STUDENTS IN ABANCAY, PERU}

\begin{abstract}
To determine the prevalence of serological markers of viral hepatitis B in university students of the city of Abancay, we performed a cross-sectional study on 240 students from three universities, from January to October 2010. Informed consent was requested to every student, an epidemiological record was filled, and a venous blood sample was drawn to determine the presence of $\mathrm{HBsAg}$, total anti - HBcAg, anti - HBe, $\mathrm{HBeAg}$ and IgM Anti - HBc by ELISA. A prevalence of $2.5 \%$ (six positive samples) was found for $\mathrm{HBsAg}$ and of $28.3 \%$ (68 positive samples) for anti - HbcAg antibodies. The male sex was associated with the presence of anti $-\mathrm{HBcAg}(\mathrm{OR}=2.0,95 \% \mathrm{Cl}, 1.2$ to 3.6). We did not found $\mathrm{HBeAg}$ or IgM anti - HBc, however, the $6 \mathrm{HBsAg}$ carriers were anti - HBe positive. In conclusion hepatitis B infection is still a public health problem in Abancay, with a significant prevalence in university students.
\end{abstract}

Key words: Seroepidemiologic Studies ; Hepatitis B; Students; Abancay; Peru (source: MesH NLM).

\section{INTRODUCCIÓN}

La infección por el virus de la hepatitis B continúa siendo un problema de salud pública, de elevada mortalidad y distribución mundial, estimando en 2000 millones de personas las infectadas y, según la OMS, existen 350 millones de portadores crónicos del HBsAg y 5,5 millones de nuevos casos de infección aguda, calculándose que 520000 personas mueren por causas asociadas a la infección, cirrosis hepática y hepatocarcinoma (1). Debido a los distintos patrones de distribución geográfica del VHB en el mundo, el 45 $\%$ de la población mundial vive en regiones de alta prevalencia (donde más del $8 \%$ es HBsAg positivo), $43 \%$ vive en regiones de prevalencia intermedia (2-7 \% con HBsAg positivo) y $12 \%$ en áreas de baja prevalencia (menor al $2 \%$ son portadores del HBsAg) (2).

\footnotetext{
Facultad de Ciencias Biológicas, Universidad Nacional de San Antonio Abad del Cusco. Cusco, Perú.

Hospital Regional Guillermo Díaz De La Vega. Apurímac, Perú.

Laboratorio Referencial, DIRESA-Apurímac. Apurímac, Perú.

a Biólogo

* Este estudio fue presentado y sustentado como tesis para optar el título profesional de Biólogo en la Facultad de Ciencias Biológicas de la Universidad Nacional de San Antonio Abad del Cusco en marzo de 2011.
} 
En Latinoamérica, la prevalencia para el HBsAg es alta en la cuenca Amazónica entre Brasil, Colombia, Venezuela y Perú; intermedia en Guatemala, Honduras y Cuba, y baja en Chile, México, Argentina y Uruguay ${ }^{(3)}$.

El Perú es considerado como área de endemicidad intermedia para el $\mathrm{VHB}$ con prevalencias promedio de $1-2 \%$ para el HBsAg y $20-30 \%$ para anti-HBcAg ${ }^{(4)}$. No obstante tiene zonas de alta, baja e intermedia endemicidad, debido a la intensa migración que caracteriza a nuestro país ${ }^{(5)}$. Así mismo existen áreas hiperendémicas en la cuenca de la Amazonía peruana con tasas de portadores del HBsAg de 9,8 \% y $59 \%$ para anti-HBcAg ${ }^{(6)}$, mientras que en la sierra peruana algunas valles interandinos de la vertiente oriental de la cordillera de los andes como Abancay y Huanta son consideradas regiones hiperendémicas por las altas tasas de portadores del HBsAg ${ }^{(7,8)}$.

Estudios previos en la ciudad de Abancay confirmaron la hiperendemicidad de la infección por VHB, en 2009 se halló una prevalencia de 1,9 \% para el HBsAg y 35,3\% para anti-HBcAg en donantes del banco sangre del Hospital Regional de Abancay ${ }^{(9)}$. Sin embargo, no hay más estudios en la población general. Por ello, nuestro estudio tiene como objetivo conocer la prevalencia de la infección por el VHB en una población de estudiantes universitarios, aparentemente sanos, de la ciudad de Abancay.

\section{EL ESTUDIO}

Se realizó un estudio transversal y descriptivo, entre enero a octubre de 2010. La población estuvo conformada por 5261 estudiantes de tres universidades de Abancay: Universidad Tecnológica de los Andes (UTEA), Universidad Alas Peruanas filial Abancay (UAP-Abancay) y Universidad Micaela Bastidas de Apurímac (UNAMBA), donde se realizaron campañas de despistaje de la infección por VHB; por la facilidad del caso se seleccionó un tamaño muestral de 240 estudiantes que desearon participar voluntariamente en el estudio. El método de muestreo usado fue no probabilístico por conveniencia; donde se incluyó a estudiantes de 18 años a más, aparentemente sanos, que residían en Abancay por cinco o más años.

\section{TÉCNICAS E INSTRUMENTOS DE RECOLECCIÓN DE LA INFORMACIÓN}

El estudio fue revisado y aprobado por la Facultad de Ciencias Biológicas de la Universidad Nacional de San Antonio Abad del Cusco. Previa coordinación se ingresó a las universidades a promover charlas de sensibilización entre los estudiantes sobre la transmisión de la infección y secuelas de la enfermedad, detallando los objetivos y beneficios del estudio. Todos los participantes firmaron un consentimiento informado, luego procedieron a llenar una ficha epidemiológica que indagaba sobre datos demográficos, antecedentes de la enfermedad y factores de riesgo para la infección.

A cada participante se le extrajo una muestra de $5 \mathrm{~mL}$ de sangre por venopunción en la región ante-cubital, dichas muestras fueron llevadas al Banco de Sangre del Hospital Guillermo Díaz de La Vega, para la obtención de suero sanguíneo por centrifugación para luego determinar HBsAg y Anti-HBcAg por la técnica de ELISA (Biokit color) y detección de los anti-HBe (Labaustria). Las muestras seropositivas al HBsAg fueron enviadas al INS para su confirmación y detección de HBeAg.

\section{ANÁLISIS ESTADÍSTICO}

La información obtenida se ingresó y se procesó en SPSS versión 15.0 para Windows; para la elaboración de las tablas se empleó el programa Excel 2010. Se calculó las frecuencias y porcentajes para variables discretas. Para las variables continuas se calculó la media y la desviación estándar. Mediante el análisis bivariado a través de chi cuadrado, se calculó la asociación estadística tomando como significativo un $p<0,05$. Para cuantificar la fuerza de asociación se calculó el odds ratio (OR) con un intervalo de confianza de $95 \%$.

\section{HALLAZGOS}

Un total de 240 estudiantes participaron del estudio, la edad promedio fue de $24,1 \pm 4,1$ años. El 91,3\% tenían una edad comprendida entre 18 a 27 años y 143 (60\%) fueron mujeres. Se identificaron seis casos seropositivos

Tabla 1. Características demográficas y seroprevalencia del HBsAg, anti-HBc en estudiantes universitarios de Abancay, Perú. 2010

\begin{tabular}{lccr}
\hline & & Frecuencia & ( \%) \\
\hline Universidad & UTEA & 80 & 33,3 \\
& UAP & 79 & 32,9 \\
Sexo & UNAMBA & 81 & 33,8 \\
& Masculino & 97 & 40 \\
Edad & Femenino & 143 & 60 \\
& $18-27$ & 219 & 91,3 \\
& $28-37$ & 17 & 7,1 \\
Seroprevalencia & $>37$ & 4 & 1,7 \\
& HBsAg & 6 & 2,5 \\
\hline
\end{tabular}

UTEA: Universidad Tecnológica de los Andes

UAP: Universidad Alas Peruanas Filial Abancay

UNAMBA: Universidad Micaela Bastidas de Apurímac 
Tabla 2. Prevalencia de marcadores $\mathrm{HBsAg}$, anti-HBcAg para el VHB según sexo en estudiantes universitarios de Abancay, Perú. 2010.

\begin{tabular}{lcccc}
\hline & \multicolumn{2}{c}{ Sexo } & & \\
\cline { 2 - 4 } $\begin{array}{c}\text { Marcador } \\
\text { Serológico }\end{array}$ & $\begin{array}{c}\text { Masculino } \\
(\mathrm{n}=97)\end{array}$ & $\begin{array}{c}\text { Femenino } \\
(\mathrm{n}=143)\end{array}$ & & $\begin{array}{c}\text { OR } \\
(\mathrm{IC}=95 \%)\end{array}$ \\
\hline HBsAg & $6(6,2 \%)$ & 0 & NA & NA \\
Anti-HBcAg & $36(37,1 \%)$ & $32(22,4 \%)$ & 0,013 & $2,0(1,2-3,6)$ \\
\hline
\end{tabular}

p y OR: obtenidos al comparar ambos sexos.

NA: No Aplicable

al HBsAg siendo la prevalencia de 2,5\%; y 68 con infección previa por hepatitis $\mathrm{B}$, con una prevalencia de 28,3\% para los anticuerpos anti-HBcAg (Tabla 1).

El sexo masculino estuvo asociado con la presencia de anticuerpos anti-HBcAg $(p<0,013)$. Por consiguiente, tuvieron dos veces mayor probabilidad de presentar anticuerpos anti-HBcAg (OR = 2,0; IC 95 \%, 1,2-3,6) (Tabla 2).

No se encontró marcadores serológicos antígeno "e" de la hepatitis $\mathrm{B}(\mathrm{HBeAg})$ e IgM anti-HBcAg entre los seis estudiantes con serología positiva para el HBsAg. Solo se detectó la presencia de anticuerpos contra el antígeno "e" de la hepatitis B (anti-HBe) en los seis portadores del HBsAg.

Ninguno de los factores de riesgo considerados en el estudio estuvieron asociados con la infección por el VHB y la presencia de anticuerpos anti-HBcAg (Tabla 3).

\section{DISCUSIÓN}

Encontramos una prevalencia de portadores del HBsAg de $2,5 \%$ y $28,3 \%$ para los anticuerpos anti-HBcAg que son inferiores a las publicaciones de Méndez et al. ${ }^{(4)} \mathrm{e}$
Indacochea et al. ${ }^{(7)}$ que encontraron prevalencias de 14 $\%$ y $9,8 \%$ para el HBsAg, y demostraron la hiperendemicidad de la infección, posteriores estudios de Flores et al. (10) encontraron 4,0\% de portadores de HBsAg y, en 38,3 $\%$, antecedente de infección por VHB, hallazgos parecidos a los resultados de los anticuerpos anti-HBcAg $(28,4$ $\%)$ del hospital ESSALUD de Abancay (11) y diferente e inferior a los hallazgos en otros valles interandinos de la sierra como Huanta y el valle del río Pampas (Andahuaylas y Ayacuho) ${ }^{(8,12)}$. Estas cifras de prevalencia encontrados en este valle interandino, posiblemente se deben a las características ecológicas, geográficas y demográficas de la ciudad. Otra posible explicación a esta reducción sería el impacto generado por la inmunización contra el VHB que se ha integrado al Programa Ampliado de Inmunizaciones (PAI) y se realiza con regularidad desde $1991^{(13)}$.

De otro lado, algunos estudios han demostrado la asociación entre el sexo masculino y la presencia del HBsAg ${ }^{(7,14)}$. Nuestros hallazgos muestran que el sexo masculino estuvo asociado con la presencia de anticuerpos anti-HBcAg.

Todos los portadores del HBsAg eran portadores crónicos de baja infecciosidad. Tales condiciones coinciden con otros estudios nacionales donde no encontraron IgM anti-HBcAg en portadores del HBsAg ${ }^{(15)}$. Sin embrago, estos hallazgos difieren de los estudios de zonas hiperendémicas como la cuenca amazónica y el valle del río Pampas donde encontraron portadores del $\mathrm{HBsAg} / \mathrm{HBeAg}(+)$ que son considerados potencialmente infecciosos para la transmisión del VHB ${ }^{(6,12)}$.

No se pudo determinar los factores de riesgo en la población universitaria, ello podría deberse a que en Abancay existen otras vías de transmisión poco conocidas como la transmisión vertical o la presencia de posibles insectos hematófagos que actúan como vectores

Tabla 3. Factores de riesgo evaluados en estudiantes universitarios de Abancay, Perú. 2010.

\begin{tabular}{lrrrrr}
\hline Factor de riesgo & Población & Anti-HBcAg (+) & OR & IC (95 \%) & $\boldsymbol{p}$ \\
\hline Transfusión sanguínea & $9(3,8)$ & $1(1,5 \%)$ & 0,3 & $0,04-2,49$ & 0,24 \\
Uso de inyectables / EV & $142(59,2)$ & $38(55,9 \%)$ & 0,8 & $0,46-1,46$ & 0,52 \\
Atención dental & $175(72,9)$ & $47(69,1 \%)$ & 0,8 & $0,41-1,42$ & 0,41 \\
Cirugías & $34(14,2)$ & $14(20,6 \%)$ & 0,5 & $0,23-1,05$ & 0,07 \\
Tatuajes / piercing & $10(4,2)$ & $3(4,4 \%)$ & 1,1 & $0,27-4,33$ & 0,91 \\
Conducta sexual de riesgo & $57(23,8)$ & $16(23,5 \%)$ & 0,9 & $0,50-1,90$ & 0,96 \\
Antecedente intrafamiliar & $50(20,8)$ & $16(23,5 \%)$ & 1,2 & $0,63-2,45$ & 0,52 \\
Antecedente de ictericia & $9(3,8)$ & $5(7,4 \%)$ & 0,3 & $0,07-1,07$ & 0,06 \\
Familiar fallecido con enfermedad hepática & $3(1,3)$ & $3(4,4 \%)$ & NA & NA & NA \\
Acupuntura & $2(0,8)$ & & NA & NA \\
\hline
\end{tabular}

p y OR: al comparar la presencia de anti-HBcAg y los factores de riesgo.

NA: No Aplicable 
mecánicos, entre ellos la "chirimacha" (Triatoma infestans) (16) por la diversidad ecológica y geográfica de la ciudad, lo cual favorecería el desarrollo del posible vector. Otra limitación para determinar esta relación sería el pequeño tamaño muestral analizado y la estrategia de muestreo.

Recomendamos realizar en Abancay, el seguimiento de los portadores crónicos de VHB con la presencia de IgM anti-HBcAg, HBeAg, anti-HBe, vigilar sus posibles complicaciones y evaluar la transmisión horizontal e intrafamiliar de los portadores crónicos. Además, es necesario realizar un estudio que permita evaluar el impacto de la vacunación.

Las cifras de prevalencia encontradas aparentemente son inferiores a los anteriores informes en Abancay $(4,7,10)$ por ello, es importante señalar que el presente estudio tiene varias limitaciones, como el pequeño tamaño muestral que no es representativo de la población estudiada; de otro lado, la estrategia de muestreo generó una predominancia en mujeres (60 \%) y la prevalencia de portadores es predominante en hombres; por tanto, la prevalencia de portadores y de infección encontradas puede ser menor a la real. Este hallazgo debe confirmarse en una población más grande que respaldaría su validez y seria un aporte consistente a la investigación epidemiología de la infección.

En conclusión, la prevalencia de hepatitis B en estudiantes universitarios de Abancay es alta, confirmando el problema que genera en salud pública. Esperemos que nuestro estudio contribuya al conocimiento de la epidemiología de esta enfermedad en la población de estudiantes mayores de 18 años y a futuras investigaciones en áreas endémicas para determinar los factores de riesgo.

\section{AGRADECIMIENTOS}

Agradecimientos especiales a todo el personal profesional y técnico de Servicio de Patología y Banco de Sangre del Hospital Guillermo Díaz de la Vega, a los doctores Edison Vivanco Quinte, Yaneth Mirian Apac Robles y al médico Jaime Vargas Flores.

\section{Contribuciones de autoría}

MCRS, MHA y EGAA participaron en la concepción y diseño del estudio, MCRS MHA y JPQO contribuyeron en recolección y obtención de los resultados, el análisis e interpretación de los datos fue realizado por MCRS y EGAA; MHA y MCRS participaron en la redacción del manuscrito y aportaron material de estudio. Todos los autores revisaron en forma crítica las versiones preliminares del manuscrito y aprobaron la versión final del trabajo.

\section{Fuentes de financiamiento}

El estudio en mención contó con el apoyo técnico-financiero del Hospital Regional Guillermo Díaz De La Vega de Abancay, Laboratorio Referencial de salud Pública de la DIRESA-Apurímac y Municipalidad Provincial de Abancay.

\section{Conflictos de interés}

Los autores declaran no tener conflicto de interés en la publicación del presente artículo.

\section{REFERENCIAS BIBLIOGRÁFÍCAS}

1. Lavanchy D. Hepatitis B epidemiology, disease burden, treatment and current and emerging prevention and control measures. J Viral Hepat. 2004;11(2):97-107.

2. Alter MJ. Epidemiology and prevention of hepatitis B. S Liver Dis. 2003;23:39-46.

3. Cubides V, Suárez C, Quintero P. Epidemiología e historia natural de la hepatitis B. Rev Col Gastroenterol. 2009;24(1):4-12.

4. Méndez $M$, Arce $M$, Kruger, Sánchez $S$. Prevalencia de marcadores serológicos de hepatitis vírica en diversos grupos de población del Perú. Bol Of Panam. 1989;106(2):127-38.

5. Cabezas C. Hepatitis viral B y delta: epidemiología y bases para su control. Lima: Ministerio de Salud del Perú; 2008.

6. Cabezas C, Suárez M, Romero G, Carrillo C, García M, Reátegui J, et al. Hiperendemicidad de hepatitis viral B y Delta en pueblos indígenas de la Amazonía peruana. Rev Peru Med Exp Salud Publica. 2006;23(2):114-22.

7. Indacochea S, Gotuzzo E. De La Fuente J, Phillips I, Whignal S. Elevada Prevalencia de marcadores de hepatitis B y Delta en el valle interandino de Abancay. Rev Med Hered. 1991;2(4):168-72.

8. Cabezas C, Gotuzzo E, Phillips E. Prevalencia de marcadores serológicos de hepatitis viral A, B y Delta en escolares aparentemente sanos de Huanta (Perú). Rev Gastroenterol Peru. 1994;14(2):123-34.

9. Hospital Regional Guillermo Díaz De La Vega, Servicio de Banco de Sangre. Informe de registro de donantes 2000 - 2009. Abancay: Hospital Regional Guillermo Díaz De La Vega; 2009.

10. Flores A, Gotuzzo E, Cabezas C, Watts D, Holgado W. Estudio demográfico de la hepatitis B y Delta en la ciudad de Abancay-Apurímac. Bol Soc Per Infecc Trop. 1995;4:26

11. Dávalos E. Prevalencia de antígenos y anticuerpos de la hepatitis $B$ y hepatitis $C$ por el método (EIA) inmunoenzayo enzimático en población aparentemente sana en el hospital de apoyo I de IPSS-Abancay [Tesis Bachiller]. Cusco: Facultad de Ciencias Biológicas, UNSAAC; 1995.

12. Segovia G, Galván K, García V, Huamaní L, Gotuzzo E. Prevalencia de marcadores serológicos para hepatitis $B$ y Delta e infección Intrafamiliar en el valle del rio Pampas, Perú. Rev Peru Med Exp Salud Publica. 2002;19(2):5762. 
13. Cabezas C, Echevarría C, Gómez G, Gotuzzo E. Programa Piloto de Inmunización contra Hepatitis Viral B, integrado al Programa Ampliado de Inmunizaciones (PAI) en Abancay (Perú). Rev Gastroenterol Peru. 1995;15:21522

14. Rolando N, Figueroa R, Takano J, Guillen C, Lizárraga J, Jave J. Estudio clínico, bioquímico, y anatomopatológico de los portadores de antígeno de superficie de la hepatitis (HBsAg) en Abancay. Rev Gastroenterol Peru. 1983;3(1):22-32.

15. Cabezas C, Anaya E, Bartalesi F, Sánchez J. Transmisión horizontal de Hepatitis Viral B en población Desplazada en un área Hiperendémica a sus contactos en un área de Baja Endemicidad del Perú. Rev Gastroenterol Peru. 1997;17(2):128-34.
16. Cohen J, Mandolesi M F, Laszlo M, Illanes L. El Triatoma infestans como vector del virus de la hepatitis $\mathrm{B}$. Acta Gastroenterol Latinoam. 1981;11(1):215-23.

Correspondencia: Max Carlos Ramírez Soto

Dirección: Universidad Nacional de San Antonio Abad del Cusco. Av. de la Cultura N. ${ }^{\circ}$ 733. Cusco, Perú.

Teléfono: 984-887238

Correo electrónico: maxcrs22@gmail.com

\section{Consulte las ediciones anteriores de la} Revista Peruana de Medicina Experimental y Salud Pública en

\section{www.scielosp.org}

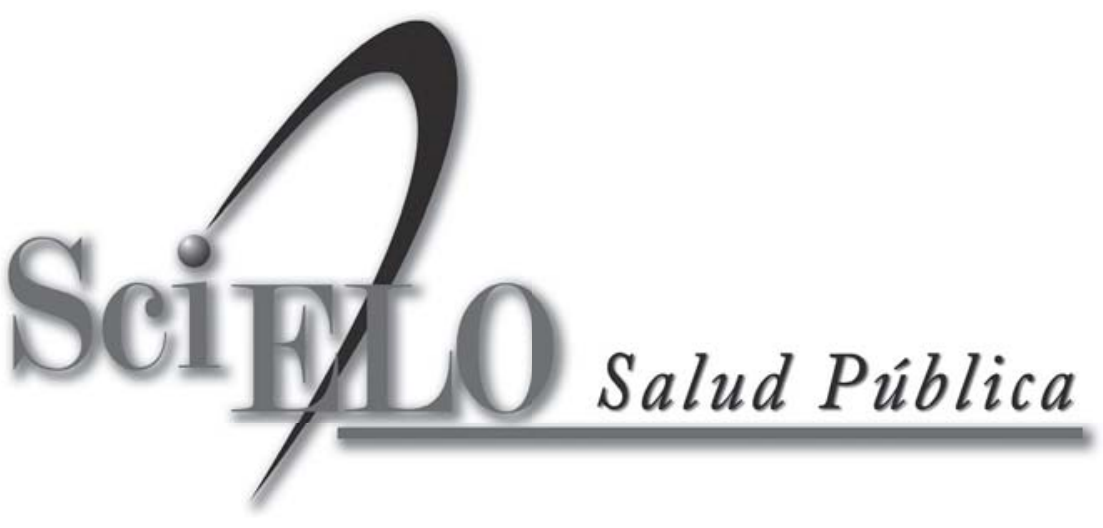

